VOLUMEN I 
“Transfer” XV: 1-2 (2020), pp. 1-11. ISSN: 1886-554

\title{
NÓs-OUTRAS. COMPORTAMENTOS E INTERVENCIÓNS TRADUTIVAS UNA REVISIÓN CRÍTICA PERMANENTE.
}

\author{
Áurea Fernández Rodríguez (ORCID: 0000-0003-4225-4925) \\ Ana Luna Alonso (ORCID: 0000-0001-8999-3077) \\ Universidade de Vigo-BITRAGA ${ }^{1}$
}

El número monográfico titulado Nós Outras. Comportamentos e intervencións tradutivas nace como un proyecto más del grupo de investigación BiTRAGA de la Facultade de Filoloxía e Tradución de la Universidade de Vigo. Se trata de la creación de un espacio de debate sobre Feminismos y Estudios de Traducción e Interpretación (ET\&I) que quiere ir más allá del campo literario, lugar en el que se han ido desarrollando tradicionalmente estos estudios. ${ }^{2}$

La propuesta centra el enfoque en el trabajo de las mujeres traductoras e intérpretes (teóricas, profesionales, agentes o responsables del proceso mediador) y en el papel que representan en la historia reciente de la traducción o la interpretación con especial atención al traslado cultural dentro del Estado español. Así, los trabajos giran alrededor de objetivos como destacar el trabajo de traductoras y/o intérpretes; revisar la historia de la traducción desde un enfoque de género y contribuir a la Historia de la T\&I con la construcción de un canon de mujeres traductoras e intérpretes femeninas y autoras traducidas en las diferentes

1 En: http://bitraga.gal. Grupo integrado en BiFeGa, financiado por la Consellería de Cultura, Educación e Ordenación Universitaria de la Xunta de Galicia en el marco del Programa de Consolidación e Estruturación de Unidades de Investigación Competitivas (2017-2019) con referencia: ED431B 2017176.

2 Las editoras se integran en el proyecto "Nuevas estrategias de promoción cultural. Las ferias internacionales del libro y la condición de invitado de honor", CULTURFIL de la USC, financiado por FEDER/Ministerio de Ciencia, Innovación y Universidades-Agencia Estatal de Investigación con referencia: FFI2017-85760-P.

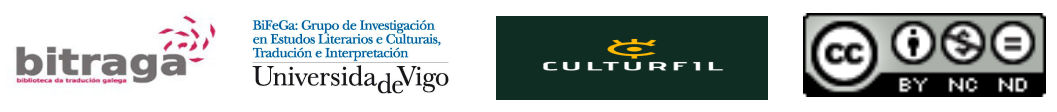


“Transfer” XV: 1-2 (2020), pp. 1-11. ISSN: 1886-554

culturas; visibilizar el mapa de editoras de obra femenina traducida y el catálogo de traductoras; ofrecer una visión de los fenómenos y tendencias más relevantes en el ámbito de estudio en el que ni el uso del lenguaje ni la transmisión de ideología son ajenos; analizar textos, condiciones laborales, políticas de traducción, líneas de actuación editorial, etc.

Con el objetivo de contribuir a desenmascarar los mecanismos de poder que subyacen a cualquier reescritura, nos interesa el proceso de resistencia cultural que supone la interacción entre feminismos y traducción, como disciplinas que cuestionan discursos hegemónicos y progresan en la reflexión crítica, cuestionándola desde el debate interno. Además, queremos destacar el trabajo de las traductoras e intérpretes en la disciplina de la T\&I y cooperar con la reflexión sobre la deontología profesional. Para poder conseguirlo, debemos revisar la historia de las áreas de conocimiento desde un enfoque de género y analizar desde esa perspectiva, quién traduce, cómo se traduce, para quién y para qué se traduce, cuál es el canon, quién lo crea, para qué y por qué.

Así, con la intención de ofrecer una visión de fenómenos y tendencias relevantes, iniciamos esta tarea gracias a la colaboración de expertas polifacéticas escritoras, editoras, mediadoras, traductoras e investigadoras como Montserrat Bacardí, Patricia Buján Otero y María Xesús Nogueira en la primera edición. A ellas se han ido sumando otras protagonistas como Nuria Brufau, Carmen Francí, Elisabete Manterola, Celia Recarey y Laura Saéz. Más tarde se asomarían a esta ventana Olga Castro, Pilar Godayol, Eva Mejuto, María Reimóndez y José Santaemilia. Todo un elenco de expertas conscientes de que la T\&I desempeña un decisivo papel en la sociedad en general y que se erige como un arma poderosa para denunciar la naturaleza patriarcal y sexista del lenguaje.

Uno de los campos en los que la lucha por la igualdad entre mujeres y hombres ha sido crucial es el lenguaje, herramienta imprescindible para la traducción y la interpretación y un elemen-

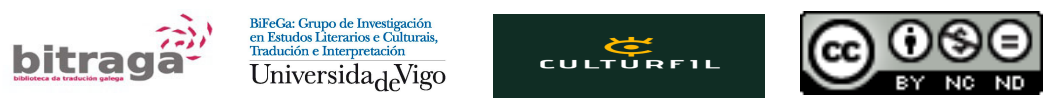


“Transfer” XV: 1-2 (2020), pp. 1-11. ISSN: 1886-554

to esencial en la transmisión y mantenimiento de ideologías. La traducción y la interpretación son actividades que influyen en la evolución de una lengua, por lo que puede perpetuar o modificar tendencias, pasar de un lenguaje patriarcal a uno consciente no sexista. La traducción no es neutra, ni invisible, ni fiel, es intervención y poder. Se trata de una disciplina en constante y necesaria transformación que debe acercar posturas entre la teoría y la práctica profesional y entre los diferentes espacios geográficos.

El monográfico centra el enfoque en la tarea de las mujeres traductoras, intérpretes y agentes mediadoras (editoras, promotoras, académicas, investigadoras, etc.) en el marco de las distintas lenguas y culturas del Estado español. Parte de lo más próximo con la intención de conocer las desigualdades y los desequilibrios generados en un espacio político-social de vecindad. Los contextos catalán, vasco y gallego deben conocerse más y mejor en un marco Ibérico que permite desvelar y comprender fenómenos en ocasiones manifiestamente ignorados. La historia de la T\&I debe incluir la historia de mujeres y hombres, la historia de muchas protagonistas silenciadas o ignoradas, y tiene como compromiso prioritario revelar las razones de dicha ocultación.

En un mismo escenario historiográfico se nos presenta la entrega de Montserrat Bacardí, quien destaca la figura de la escritora y traductora Maria Dolors Orriols (1914-2008), reescritora de su propia obra mediante la traducción y de textos vanguardistas y rompedores de Franz Kafka o Georges Bernanos. Editora en los años cincuenta de Aplec, una de las revistas culturales que ha sufrido la persecución de las autoridades franquistas. María Dolors Orriols, como muchas mujeres de su tiempo, se verá obligada a recluirse en casa, ante la prohibición de trabajar fuera. Se forma de manera autodidacta, lee mucho, escribe y tiene que viajar con el permiso marital. En Francia encontró su fuente de inspiración y refugio intelectual hasta la desaparición del dictador. El artículo de Pilar Godayol se sitúa en la transición democrática de la Barcelona de la década de los ochenta. Una

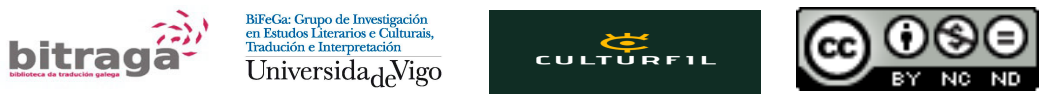


“Transfer” XV: 1-2 (2020), pp. 1-11. ISSN: 1886-554

época de apertura política y cultural muy enriquecedora en la que se forjan proyectos como el café-bar laSal y la editora homónima, la primera empresa editorial feminista del Estado. Godayol describe el catálogo de laSal, centrándose en el análisis de los textos seleccionados para ser publicados en las colecciones Ensayo (1978-1989) y Cuadernos Inacabados (1981-1988), respectivamente, en los que la traducción tuvo un papel fundamental al visibilizar discursos y tendencias del feminismo nacional e internacional. Al inicio de su trabajo, la profesora de la Universidad de Vic repasa el papel innovador de la universidad española en los últimos veinte años sobre el estudio de la intersección "género, mujer y traducción" y nos ofrece retos pendientes para la disciplina.

Impulsar políticas de traducción a favor de la visibilización de las escritoras es uno de los objetivos principales del feminismo, ${ }^{3}$ pero más allá de aumentar su presencia, es preciso reflexionar sobre el lugar y la forma en que se potencia dicha visibilización. En este sentido ofrece su propuesta de análisis la investigadora María Reimóndez. La autora nos invita a reflexionar sobre la práctica de la traducción y sobre todo de la interpretación con perspectiva ética comprometida, buscando evitar el conflicto y considerando la parte positiva de ser una traductora feminista. Para Reimóndez hay aquí un campo de estudio por descubrir. La investigadora conoce desde dentro el mercado, tanto el local como el internacional, y su enfoque quiere relacionar los estudios sobre el estado real de la profesión con el modo en que la perspectiva feminista puede y debe ayudar a mejorarla.

Desde el espacio profesional e investigador, dos artículos, uno de Carmen Francí y otro de José Santaemilia, centran el inte-

\footnotetext{
${ }^{3}$ Durante el lustro 2014-2018, únicamente una séptima parte de las obras españolas que recibieron una subvención a la traducción ha sido firmada por mujeres. Este desequilibrio no favorece la visibilización de las autoras e impide una sostenibilidad cultural que debe ser socialmente transversal (Montero Küpper 2019).
}

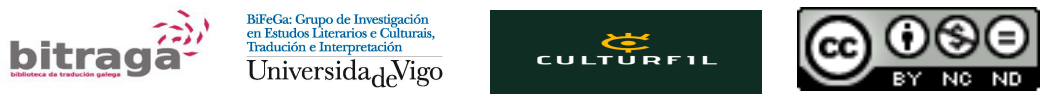


“Transfer” XV: 1-2 (2020), pp. 1-11. ISSN: 1886-554

rés en el debate sobre la presencia de las mujeres en el mundo de la traducción profesional, sobre todo en la traducción literaria o editorial.

Francí señala que la profesión tiene un peso importante en los beneficios de la industria editorial dentro del Estado español. Esta actividad es reivindicada por la autora del artículo que como buena conocedora de la problemática del colectivo considera cada vez más explotada. Realiza un análisis cualitativo a partir de los datos y declaraciones de las personas que trabajan desde dentro en el sector y presenta algunas de las explicaciones de la feminización que no es equivalente, ni mucho menos sinónimo, de igualdad. La negociación de la persona que edita con la persona que traduce merece una atenta lectura sobre cuáles son las condiciones en las que se desarrolla la labor de la profesional, es decir, si tiende a ocupar una posición subordinada, si traduce obras y géneros menos formales o si tiene menos presencia en lenguas centrales.

Santaemilia, por su parte, repasa los informes elaborados hasta el momento y valora a partir de los datos las consecuencias que tiene para las mujeres en el mundo de la traducción la "feminización". En este trabajo confirma que la "feminización" de la educación no se traslada en igual volumen a la ocupación profesional, que esconde una real brecha de género y un manifiesto techo de cristal. El autor aborda la cultura laboral patriarcal que lleva a las mujeres a ocupar espacios de subordinación y ofrece cifras sobre la presencia de la mujer en el ámbito de la traducción, y en el de la traducción editorial donde "se observa una sexualización de la profesión, una atribución de rasgos de género a una profesión". En este sentido, además de la necesidad de luchar contra el estereotipo, se constata que la mujer, así como el espacio lingüístico-cultural de procedencia, posee todavía menor visibilidad y reconocimiento profesional.

Elizabete Manterola examina en su aportación la interacción entre feminismo, traducción y lengua vasca constatando,

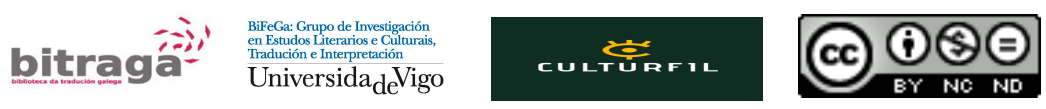


“Transfer” XV: 1-2 (2020), pp. 1-11. ISSN: 1886-554

tanto desde el punto de vista práctico como teórico, que la realidad ha cambiado y está en constante evolución. En la actualidad, hay mayor presencia de mujeres traductoras en el colectivo EIZIE y mayor presencia de la autoría femenina en los catálogos, aunque el análisis lleva a Manterola a comprobar que hay que seguir impulsando la paridad y reflexionar sobre el lugar y la forma en que se potencia la visibilización de la mujer, porque los traductores han traducido más, han recibido más premios y se han reservado el espacio del ensayo y la literatura destinada a público adulto. Del mismo modo, los estudios académicos (y los de fuera de la academia) sobre la lengua, la literatura y la traducción con perspectiva de género han ido tomando el relevo con respecto a enfoques previos en los que no se cuestionaba la necesidad de incluir el pensamiento feminista. La investigadora termina su exposición con un repaso sobre los trabajos realizados alrededor de la traducibilidad del género y las relaciones asimétricas de poder que pasan por el uso de la lengua.

Con la intención de contribuir a la historia de la traducción, Olga Castro construye un canon de autoras traducidas hacia el inglés británico, y en concreto, centra su interés en la importación de obra escrita por mujeres desde una cultura minorizada como la gallega. Castro apunta un creciente interés del público lector por autorías europeas e indaga el caso de las autorías gallegas. En sus conclusiones indica que sería necesario mejorar las estrategias de visibilización de la literatura gallega, con traducciones de fragmentos de obras que tengan potencial atractivo para la traducción recopiladas en catálogos de derechos. Además, insiste en que es crucial una mejor difusión de las ayudas a la traducción y un cambio fundamental en los trámites que hay que llevar a cabo para tramitar las solicitudes, de modo que las empresas extranjeras (normalmente independientes) puedan solicitarlas (Montero Küpper 2019).

La experiencia traductora y editora de Patricia Buján Otero acredita el riguroso análisis realizado con el catálogo de obras de

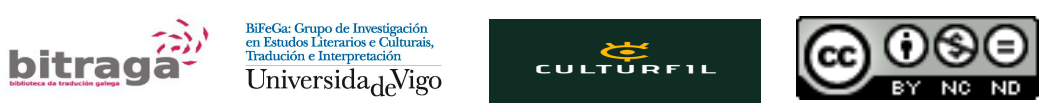


“Transfer” XV: 1-2 (2020), pp. 1-11. ISSN: 1886-554

autoras publicadas hacia la lengua gallega en la última década con la intención de observar si algo está cambiando. Es decir, investiga sobre la posibilidad de que exista una transformación en el sistema literario de importación con respecto a lo que denomina "triple marginalidad" (traducción, género y lengua), un fenómeno al que se ven sometidas las publicaciones de traducciones de obras originales escritas por mujeres. Buján reclama mayor implicación por parte de los agentes del campo literario (tanto en producción como en crítica) a la hora de proponer un canon de traducción que contribuya a configurar un nuevo sistema de valores, un corpus textual teórico y referencial de alteridad en gallego que permita acelerar el cambio que parece advertirse en los últimos tiempos.

El trabajo de Laura Sáez cierra un proyecto que abre nuevas puertas a la investigación. En su contribución se aproxima al desarrollo de una apuesta editorial de libro infantil traducido que la propia autora y traductora dirige. Su aportación a este volumen monográfico analiza el catálogo de Patasdepeixe desde su reciente creación, a principios de siglo XXI. Sáez describe con perspectiva crítica cómo ha evolucionado el fondo editorial, tanto desde el aspecto formal como la selección de las autorías, ilustraciones, etc. Al mismo tiempo, el artículo muestra de forma tangencial la realidad del sector editorial en Galicia desde una perspectiva feminista y empresarial.

Así, desde Galicia, nos atrae saber cómo se explican y cómo se impulsan los diferentes proyectos en los que de modo directo o indirecto participan las responsables de las editoras (políticas, catálogos, carencias, necesidades, gestión, etc.), que parten de una perspectiva de género en el panorama de la selección de los textos y autorías, comportamientos o aspectos profesionales. Algunos de los textos aquí presentados ofrecen las líneas de actuación de esas empresas independientes, alternativas, a veces dirigidas por mujeres que traducen y que procuran salir de los circuitos comerciales más convencionales para publicar textos que cubran espacios,

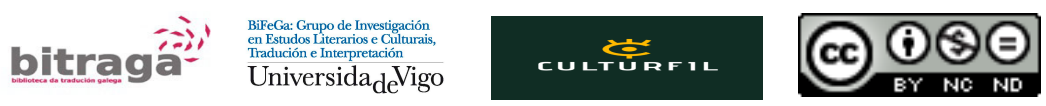


“Transfer” XV: 1-2 (2020), pp. 1-11. ISSN: 1886-554

hasta ahora desconocidos por una gran mayoría del público lector.

Como decíamos más arriba, al grupo de investigación BITRAGA ${ }^{4}$ le interesa examinar el poder legitimador de la traducción para cualquier sistema cultural, pero sobre todo para uno minoritario y minorizado como el gallego, que se manifiesta en diferentes fases, sobre todo a partir del primer tercio del siglo XX. A partir de 1975, los cambios políticos implican un importante movimiento de recuperación lingüística y cultural, que también puso en evidencia los enormes obstáculos a la internacionalización de una cultura minorizada (Galanes Santos 2018). Los primeros años de la democracia son una de las etapas más productivas en las que se analiza la contribución de las traductoras a la hora de interpretar los textos (Luna Alonso 2017). Es tiempo de reconstruir la memoria colectiva, también de manera más inclusiva, que dé cuenta de las contribuciones de las mujeres en sus propias voces y desde sus múltiples posiciones, también como lectoras (Pena Presas 2018: 10). Sirvan de ejemplo nombres como Xohana Torres (1931-2016), traductora de literatura infantil y autora del primer libro gallego traducido a las cuatro lenguas oficiales de la península (Polo mar van as sardiñas, Galaxia, 1968) (Domínguez 2008: 25); o Teresa Barros, traductora, junto con Fernando Pérez Barreiro, de Alice in Wonderland al gallego y Premio Nacional de Traducción en 1985. A estas pioneras hay que añadir muchas otras (Castro 2011) como Ursula Heinze, Luz Pozo Garza, María Xosé Queizán o Xela Arias hasta llegar al espacio más contemporáneo en el que es difícil citar todos los trabajos y reflexiones de autorías como Marilar Aleixandre, Kathleen March, Chus Pato, Erin Moure, Manuela Palacios, María do Cebreiro, Olga Castro o María Reimóndez, entre otras.

La traducción constituyó un punto de entrada en el mundo literario para muchas mujeres, ocultas en la indiferencia patriarcal que no les permitía ser autoras. Así, su actividad traductora se

${ }^{4}$ En: <<http://bitraga.gal $>>$ [Último acceso: 30/05/2019].

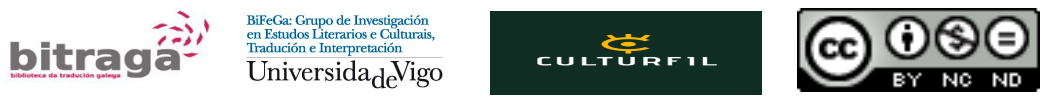


“Transfer” XV: 1-2 (2020), pp. 1-11. ISSN: 1886-554

convertiría en una de las formas de expresión pública con la que salir del anonimato y la marginación. En un espacio como Galicia, en el que Rosalía de Castro es la mejor representante para el sistema literario gallego, traductora feminista y precursora sin precedentes (Reimóndez 2017: 40), resulta especialmente significativa la ausencia de trabajos sobre la visibilización de las traductoras o la ausencia de escritoras traducidas en la actualidad hacia espacios geográficos hegemónicos o no, quizás por falta de reconocimiento o promoción interna, en comparación con las aportaciones de las traductoras en otros territorios con lengua propia dentro del Estado (Fernández Rodríguez 2014a y 2014b).

Así lo demuestran las estudiosas que participan en este número especial. Todo un lujo de contribuciones. Los trabajos dialogan entre ellos en un mismo marco espacial y temporal donde se quiere recuperar también las ventajas de no situarse en un sistema no hegemónico (Arruza, Bhattacharya e Fraser 2019). La monografía se sitúa en un movimiento creciente de reflexión teórica y práctica en el que feminismo, traducción y lengua interaccionan. El volumen ofrece datos recientes que hacen hincapié en las personas que traducen e interpretan, tratan de sus condiciones laborales, de las políticas de ayudas públicas o privadas y sobre todo abre ventanas a la reflexión y al estudio crítico transformador. Coincidimos con Santaemilia en que "La traducción constituye, para los grupos humanos o los individuos en posición subordinada, un poderoso medio de representación, de afirmación de su identidad y, por tanto, de lucha por una igualdad efectiva" (Santaemilia 2017: 2). Esperamos que este número monográfico sirva de recurso teórico y práctico, que ayude a incorporar la transversalidad en la formación de T\&I y contribuya en la transmisión de valores inclusivos, diversos e igualitarios.

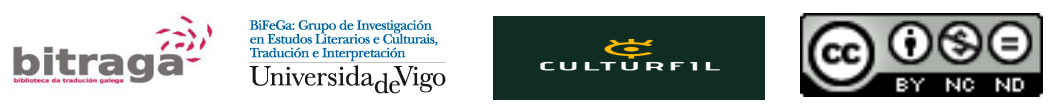


“Transfer” XV: 1-2 (2020), pp. 1-11. ISSN: 1886-554

\section{Referencias bibliográficas}

ARRUZA, Cinzia; BHATTACHARYA, Tithi \& Nancy FRASER. (2019). Feminismo para o 99\%. Un manifesto. [Trad. Patricia Buján. Limiar María Reimóndez]. Catroventos Editora.

CASTRO, Olga. (2011). "Traductoras gallegas del siglo XX. Reescribiendo la historia de la traducción desde el género y la nación", MonTI. Monografías de Traducción e Interpretación, 3: 107-130.

DOMÍNGUEZ, Mónica. (2008) "Polo mar van as sardiñas y sus traducciones". Madrygal, 11: 25-33.

---. (2009). "As primeiras traducións de literatura infantil e xuvenil ao galego: normas de tradución, difusión e recepción”. Boletín Galego de Literatura, 39-40: 37-60.

FERNÁNDEZ RODRÍGUEZ, Áurea. (2014a). "Rosalía de Castro y la traducción". Transfer, IX (1-2): 1-17

---. (2014b). "Rosalía de Castro: no principio foi a tradución". En: ÁLVÁREZ, Rosario; ANGUEIRA, Anxo; RÁBADE, Maria do Cebreiro; VILAVEDRA, Dolores. (coords.) Rosalía de Castro no século XXI. Unha nova ollada, Santiago de Compostela, Consello da Cultura Galega, 687-705.

GALANES SANTOS, Iolanda. (2018). "A proxección exterior da literatura galega e Carlos Casares: obstáculos e estratexias”, Boletín Galego de Literatura, 53(1): 31-81.

LUNA ALONSO, Ana. (2017). "O papel da tradutora no campo literario galego". Madrygal, 20: 147-156.

MONTERO KÜPPER, Silvia. (2019). "Zur kulturellen Nachhaltigkeit durch Übersetzungsförderung in Spanien”. En: Actas del I Congreso Internacional «Traducción y Sostenibilidad Cultural», Salamanca, noviembre 2018 (en prensa).

PENA PRESAS, Montse. (2018). Feminismos e literatura infantil e xuvenil en Galicia. Santiago de Compostela: Laiovento.

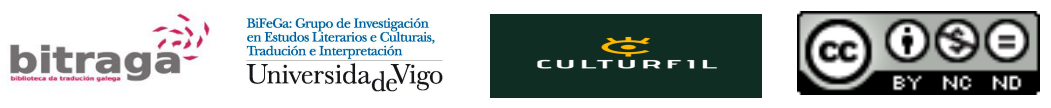


“Transfer” XV: 1-2 (2020), pp. 1-11. ISSN: 1886-554

REIMÓNDEZ MEILÁN, María. (2017). Corpos exorbitantes. Rosalía de Castro, tradutora feminista, en diálogo con Erín Moure. Santiago de Compostela: Concello de Santiago.

SANTAEMILIA, Jose. (2017). Traducir para la igualdad sexual. Translating for Sexual Equality. Granada: Comares. 